\title{
A NOTE ON HURWITZ'S ZETA-FUNCTION
}

\section{R. BALASUBRAMANIAN}

The aim of this paper is to prove an asymptotic formula for the mean square of Hurwitz's zeta-function, defined by

$$
\zeta(s ; \alpha)=\sum_{n=0}^{\infty} \frac{1}{(n+\alpha)^{s}} \quad \text { in } \quad \operatorname{Re} s>1
$$

and its analytic continuation in $\operatorname{Re} s \leqq 1$. The result is as follows:

Theorem. If $\zeta_{1}(s ; \alpha)=\zeta(s ; \alpha)-1 / \alpha^{s}$, then for $t \geqq 30$,

$$
\int_{0}^{1}\left|\zeta_{1}(1 / 2+i t, \alpha)\right|^{2} d \alpha=\log t+O(\log \log t)
$$

This improves the result of Koksma and Lekkerkerker [2] which states that

$$
\int_{0}^{1}\left|\zeta_{1}(1 / 2+i t, \alpha)\right|^{2} d \alpha=O(\log t)
$$

It is very likely that the error term $O(\log \log t)$ of the theorem can be improved.

A modification of our proof gives an asymptotic formula for $\sum_{\chi}|L(s, \chi)|^{2}$, which improves a theorem of Gallagher [1] in some range of $t$. It will form the subject matter of another paper.

We prove the theorem by establishing six lemmas.

Lemma 1. We have

$$
\zeta_{1}(1 / 2+i t, \alpha)=\sum_{1 \leqq n \leqq T} \frac{1}{(n+\alpha)^{1 / 2+i t}}+O\left(t^{-1 / 2}\right),
$$

where $T$ is the nearest integer to $t$.

Proof. This is well known. 
Lemma 2. We have

where

$$
\int_{0}^{1}\left|\zeta_{1}(1 / 2+i t, \alpha)\right|^{2} d \alpha=\log t+O\left(\left|J_{1}+J_{2}+J_{3}-J_{4}-J_{5}-J_{6}\right|\right)+O(1),
$$

$$
\begin{aligned}
& J_{1}=\sum_{k \leqq T^{1 / 2}} \frac{(T-k+1)^{1 / 2}(T+1)^{1 / 2} e^{-i t(\log (T-k+1)-\log (T+1))}}{t k}, \\
& J_{2}=\sum_{T^{1 / 2}<k \leqq T^{1 / 2} \log ^{3} T} \frac{(T-k+1)^{1 / 2}(T+1)^{1 / 2} e^{-i t(\log (T-k+1)-\log (T+1))}}{t k}, \\
& J_{3}=\sum_{T^{1 / 2} \log ^{3} T<k \leqq T} \frac{(T-k+1)^{1 / 2}(T+1)^{1 / 2} e^{-i t(\log (T-k+1)-\log (T+1))}}{t k}, \\
& J_{4}=\sum_{k=1}^{T} \frac{(1+k)^{1 / 2} e^{i t \log (1+k)}}{t k} d v, \\
& J_{5}=\frac{1}{2} \sum_{k=1}^{T} \int_{1}^{T-k+1} \frac{v^{-1 / 2}(v+k)^{1 / 2} e^{-i t(\log v-\log (v+k))}}{t k}
\end{aligned}
$$

and

$$
J_{6}=\frac{1}{2} \sum_{k=1}^{T} \int_{1}^{T-k+1} \frac{(v+k)^{-1 / 2} v^{1 / 2} \cdot e^{-i t(\log v-\log (v+k))}}{t k} d v
$$

Proof. Using Lemma 1, we have

$$
\int_{0}^{1}\left|\zeta_{1}(1 / 2+i t, \alpha)\right|^{2} d \alpha=\int_{0}^{1} \sum_{n \leqq T} \sum_{m \leqq T} \frac{1}{(n+\alpha)^{1 / 2+i t}} \frac{1}{(m+\alpha)^{1 / 2-i t}} d \alpha+O(1)
$$

and the terms corresponding to $m=n$ give the main term $\log t$, with an error $O(1)$. The terms $m \neq n$ give

$$
\begin{aligned}
& \int_{0}^{1} \sum_{n \leqq T} \sum_{m \leqq T} \frac{1}{(n+\alpha)^{1 / 2+i t}} \frac{1}{(m+\alpha)^{1 / 2-i t}} d \alpha \\
= & \int_{0}^{1} \sum_{m \leqq T} \sum_{n<m} \frac{1}{(n+\alpha)^{1 / 2+i t}} \frac{1}{(m+\alpha)^{1 / 2-i t}} d \alpha \\
+ & \int_{0}^{1} \sum_{m \leqq T} \sum_{n>m} \frac{1}{(n+\alpha)^{1 / 2+i t}} \frac{1}{(m+\alpha)^{1 / 2-i t}} d \alpha .
\end{aligned}
$$


It is sufficient to consider one of the terms. We obtain

$$
\begin{aligned}
\int_{0}^{1} \sum_{m \leqq T} \sum_{n<m} \frac{1}{(n+\alpha)^{1 / 2+i t}} \frac{1}{(m+\alpha)^{1 / 2-i t}} d \alpha \\
=\sum_{k=1}^{T} \sum_{n=1}^{T-k} \int_{0}^{1} \frac{1}{(n+\alpha)^{1 / 2+i t}} \frac{1}{(n+k+\alpha)^{1 / 2-i t}} d \alpha \\
=\sum_{k=1}^{T} \sum_{n=1}^{T-k} \int_{n}^{n+1} \frac{1}{v^{1 / 2+i t}(v+k)^{1 / 2-i t}} d v \\
=\sum_{k=1}^{T} \int_{1}^{T-k+1} \frac{1}{v^{1 / 2}(v+k)^{1 / 2}} e^{-i t(\log v-\log (v+k))} d v \\
=-i \sum_{k=1}^{T} \frac{1}{k t} \int_{1}^{T-k+1} v^{1 / 2}(v+k)^{1 / 2} d\left(e^{-i t(\log v-\log (v+k))}\right) \\
=-i \sum_{k=1}^{T} \frac{1}{k t}\left[v^{1 / 2}(v+k)^{1 / 2} e^{-i t(\log v-\log (v+k))}\right]_{1}^{T-k+1} \\
+i \sum_{k=1}^{T} \frac{1}{k t} \int_{1}^{T-k+1}\left((1 / 2) v^{-1 / 2}(v+k)^{1 / 2}+(1 / 2)(v+k)^{-1 / 2} v^{1 / 2}\right) e^{-i t(\log v-\log (v+k))} d v \\
=-i\left(J_{1}+J_{2}+J_{3}-J_{4}-J_{5}-J_{6}\right) .
\end{aligned}
$$

This proves the lemma.

Lemma 3. If $J_{4}$ and $J_{2}$ are as defined in Lemma 2, then,

Proof. Trivial.

$$
J_{4}=O(1), \quad J_{2}=O(\log \log T) .
$$

Lemma 4. If $J_{5}$ and $J_{6}$ are as defined in Lemma 2, then

$$
J_{5}=O(1), \quad J_{6}=O(1) .
$$

Proof. The result follows, using Lemma 4.3 (p. 61) of Titchmarsh [3].

Lemma 5. If $J_{1}$ is as defined in Lemma 2, then $J_{1}=O(1)$.

Proof. In $J_{1}, \log (T-k+1)-\log (T+1)$ can be replaced by $-k /(T+1)$ with a small error. Hence

$$
J_{1}=\sum_{k \leqq T^{1 / 2}} \frac{(T-k+1)^{1 / 2}(T+1)^{1 / 2} \cdot e^{i t k /(T+1)}}{t k}+O(1) .
$$

Since the partial sums of $\sum_{k} e^{i t k /(T+1)}$ are bounded, the result follows from Abel's partial summation formula. 
Lemma 6. If $J_{3}$ is as defined in Lemma 2, then

$$
J_{3}=O(1) \text {. }
$$

Proof. We apply Theorem 5.9 (p. 90) of Titchmarsh [3] to get a good bound for the sums

$$
\sum_{X \leqq k \leqq Y} e^{-i t \log (T-k+1)}
$$

where $Y \leqq X$; and $T^{1 / 2} \log ^{3} T \leqq X \leqq T / 100$, and use Abel's partial summation formula to prove that

$$
\sum_{T^{1 / 2} \log ^{3} T<k \leqq T / 100} \frac{(T-k+1)^{1 / 2}(T+1)^{1 / 2} e^{-i t(\log (T-k+1)-\log (T+1))}}{t k}
$$

is $O(1)$. We observe that

$$
\sum_{T / 100<k \leqq T} \frac{(T-k+1)^{1 / 2}(T+1)^{1 / 2} e^{-i t(\log (T-k+1)-\log (T+1))}}{t k}
$$

is $O(1)$. This proves the lemma.

The theorem follows from Lemmas 2 to 6 .

Acknowledgement. The author wishes to express his thanks to Prof. K. Ramachandra for his encouragement and for checking the manuscript.

\section{References}

[1] Gallagher, P. X.: Local mean value and density estimates for Dirichlet $L$-functions. - Indag. Math. 37, 1975, 259-264.

[2] Koksma, J. F., and C. G. Lekkerkerker: A mean-value theorem for $\zeta(s, w)$. - Indag. Math. 14, 1952, 446-452.

[3] Titchmarsh, E. C.: The theory of the Riemann zeta-function. - Clarendon Press, Oxford, 1951.

Tata Institute of Fundamental Research Homi Bhabha Road

Bombay 400005

India

Received 21 February 1978 